\title{
Monitoring temporal dynamics of steady-state visual evoked potentials
}

\author{
Benjamin Wittevrongel ${ }^{1}$ and Marc M. Van Hulle ${ }^{1}$
}

\begin{abstract}
The steady-state visual evoked potential (SSVEP) is an electrophysiological response to visual stimulation at a particular frequency. Despite its widespread adoption, primarily a spectral analysis of the recorded signal is performed, and variations in its temporal dynamics are often neglected or considered undesired. In this manuscript, we describe a method for monitoring the temporal dynamics of the SSVEP, and show its efficacy for detecting modifications of the stimulation profile on a single-trial basis. The anomalies elicited by single stimulus modifications were detected with an accuracy of $88 \%$, demonstrating the reliability of the approach.
\end{abstract}

\section{INTRODUCTION}

The steady-state visual evoked potential (SSVEP) is an electrophysiological response to flickering stimulation, and has been adopted to study a broad range of pathological and psychological phenomena, visual psychophysics and brain-computer interfacing (BCI) (see [1] for a review). As it supports the accurate and rapid selection of visual targets [2] (e.g., letters [3], symbols [4], etc.), it has gained stature in the $\mathrm{BCI}$ domain as an alternative communication channel for patients with severe motorand communication disabilities [5], [6], the viability of which was demonstrated in several use cases [7], [8], [9].

To date, the vast majority of BCI studies encode each selectable target with a unique frequency [10], phase [11] or frequency-phase combination [12], and their classifiers are tailored to detect the presence of a consistent ("steady-state") oscillatory response pattern during a given trial. Although impressive results have been reported, the vast majority of the used classifiers does not account for temporal variations in the recorded signal and even considers them as a nuisance. However, detecting such variations could open the door to equipping SSVEP-BCIs with more advanced features such as the rapid detection whether a subject is gazing (or stops gazing) at any of the targets in a non-cued manner [13] (cf. asynchronous BCI) or when the subject is switching between targets.

Some studies have investigated interruptions of the flickering stimulation in an effort to develop a hybrid

*MMVH is supported by research grants received from the Financing program (PFV/10/008), an interdisciplinary research project (IDO/12/007), and an industrial research fund project (IOF/HB/12/021) of the KU Leuven, the Belgian Fund for Scientific Research - Flanders (G088314N, G0A0914N, G0A4118N), the Interuniversity Attraction Poles Programme - Belgian Science Policy (IUAP P7/11), the Flemish Regional Ministry of Education (Belgium) (GOA 10/019), and the Hercules Foundation (AKUL 043).

${ }^{1}$ Laboratory for Neuro- and Psychophysiology, KU Leuven, 3000 Leuven, Belgium. benjamin.wittevrongel@kuleuven.be
BCI combining SSVEP and P300 responses [14], [15]. The interruption of the flickering stimulation elicits an additional positive response (P300) for the gazed target, and provides additional information for the classifier. However, the stimulation interruption was only used to elicit the P300 response, and the sudden absence of the SSVEP was not explicitly used as a feature during classification.

In this work, we describe how a model based on spatiotemporal beamforming [16], [17] can be used to investigate the temporal dynamics of the SSVEP. We demonstrate how it can detect anomalies in the SSVEP response due to transient modifications in the stimulation profile (i.e., stimulation interruptions and phase reversals), and how these could be used for target selection in a BCI context.

\section{METHODS}

To investigate the proposed method, an experiment was designed and implemented in which the flickering stimulation profile was temporarily modified. Based on the collected data, a model was trained to explore whether the stimulation modifications could be detected in the temporal response dynamics. As proof-of-concept, we used the anomalies in the temporal response to decode the gazed target.

\section{A. Experimental paradigm}

The interface of the main experiment consisted of 12 circular targets arranged in a 3 by 4 design displayed on a 24-inch monitor (VIEWPixx EEG, 1920×1080 resolution at $120 \mathrm{~Hz}$ refresh rate). Each target spanned a visual angle of $4.3^{\circ}$ with an inter-target distance of $2.4^{\circ}$, and was overlaid with a character from the latin alphabet (Figure 1A). Each row was assigned a unique frequency $(11 \mathrm{~Hz}, 13 \mathrm{~Hz}$ and $15 \mathrm{~Hz}$, respectively), and all targets within each row adopted the same frequency. A trial started with a cue (i.e., one of the characters adopted a red color), and the subject was asked to visually attend this target during the subsequent trial (left panel in Figure 1A). After the subject pressed a button, the cued character regained the same white color as the other targets. One second later, all targets started to flicker at their designated frequencies using a sinusoidal stimulation profile [18]. Note that the overlaid characters did not flicker. The flickering stimulation consisted of one second of regular flickering, followed by a stimulation profile of 10 seconds during which the flickering of the individual columns were consecutively modified (see 
A

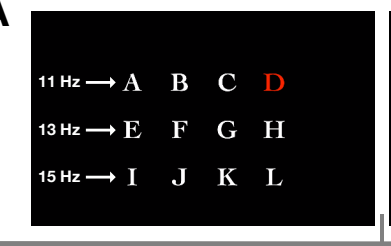

cue

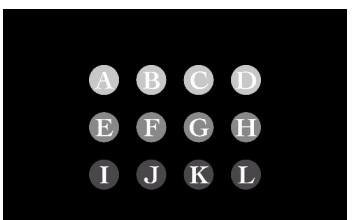

I J E L

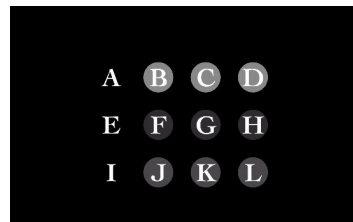

stimulation

1 second regular + non-overlapping 500 ms column modifications

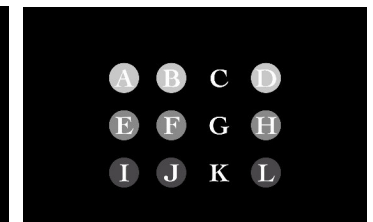

time
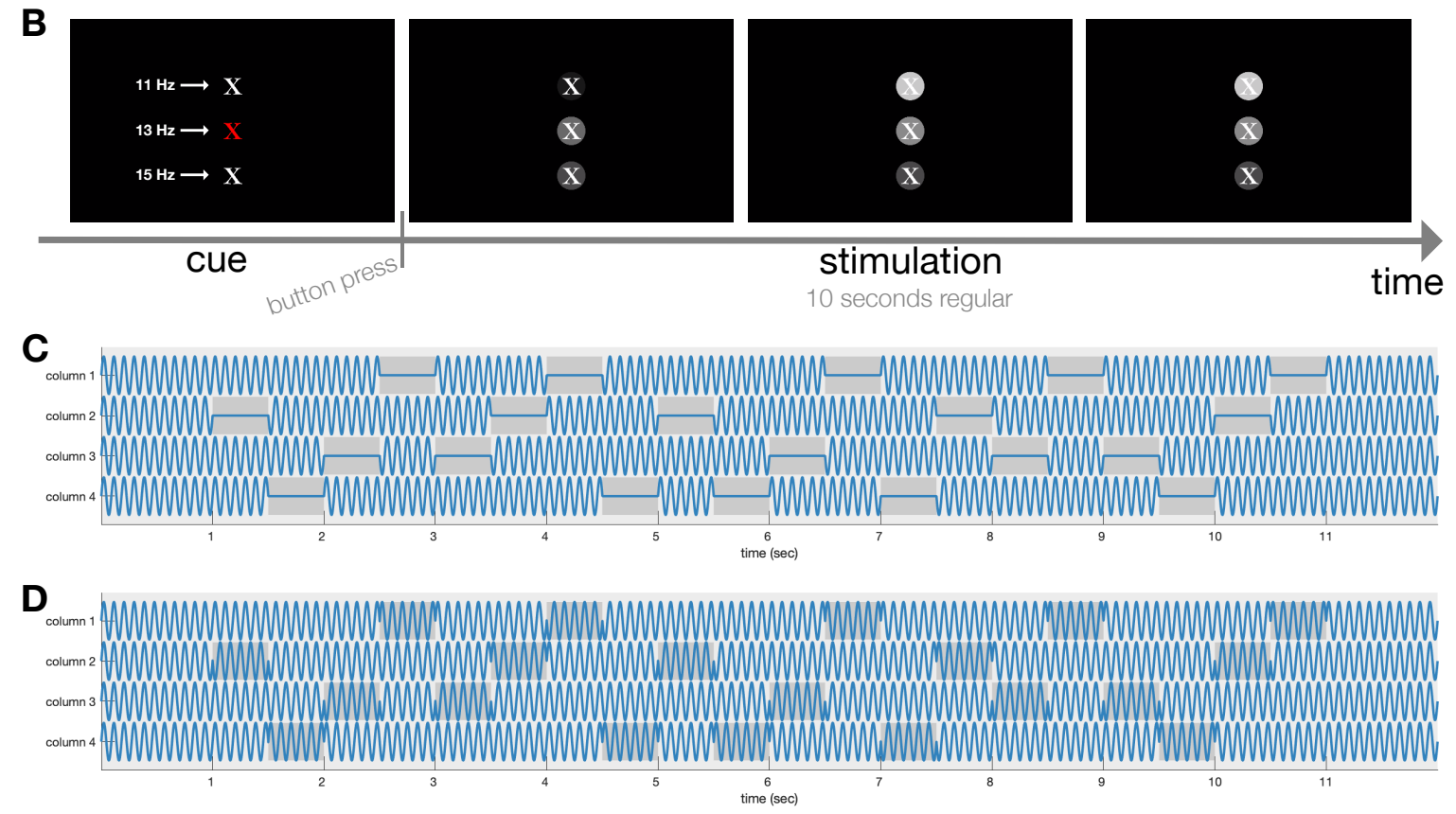

Fig. 1. Graphical representation of the experimental paradigm. (A) Interface of the main experimental session with stimulation interruptions. (B) Interface of the data collection session. (C) Luminosities (from 0 to 1 ) of the targets in the top row during one trial in the interruption session. (D) Luminosities (from 0 to 1) of the targets in the top row during one trial in the phase reversal session. The grey boxes indicate the $500 \mathrm{~ms}$ windows during which the stimulation profile was modified.

further) for 500 milliseconds, and ended with 1 second of regular flickering. During each trial, the flickering of each column was modified 5 times in pseudorandom order. Each trial lasted 12 seconds, and all characters were cued 5 times in pseudorandom order, leading to a total of 60 trials. In between trials, subjects were allowed to take a break.

The main experiment was repeated twice, each time with a different modification of the stimulation profile. In one session, the flickering stimulation was interrupted, while in the other session the flickering reversed its phase (i.e., from 0 to $\pi$ radians) during the modification period (Figure 1C and D, respectively). Each session lasted approximately 15 minutes, and all subjects participated in both sessions. Half of the subjects started with the interruption sessions, while the others performed the reversal session first.

Prior to the main experiments, an additional data collection session was performed, in which three targets, arranged in one column, were displayed (Figure 1B). The goal of this session was to collect data to train the model. The size of the targets, the inter-target distance and the assigned frequencies were equal to those of an individual column in the main experiment. Similar to the main experiment, a trial started with a cue, during which one of the characters adopted a red color, and the subject was asked to visually attend this character. After a button press, the cued character regained the white color, and one second later, the three targets started to flicker for 5 seconds at their designated frequencies using a consistent sinusoidal stimulation profile. The three targets were cued 15 times in pseudorandom order, leading to a total of 45 trials. The data collection session lasted for approximately 5 minutes.

All experiments were implemented in Matlab (2013b), using the Psychophysics Toolbox extensions [19], [20] for precise timing.

\section{B. Data acquisition}

Nine subjects ( 5 female) participated in the study. The average age was 27.1 years, ranging from 25 to 31 years old. All subjects had corrected or corrected-to-normal vision. Prior to participating, each subject was informed about the purpose of the experiment and its procedure, 
after which he/she was invited to read and, if agreed, to sign the informed consent form prior approved by the ethical committee of UZ Leuven, our university hospital.

During the experiments, EEG was continuously recorded using 8 active $\mathrm{Ag} / \mathrm{AgCl}$-electrodes, placed over the occipital scalp area, connected to a SynampsRT (Neuroscan) device operating at a sampling rate of 500 $\mathrm{Hz}$. Electrodes were placed according to the international 10-20 system with ground and reference electrodes at $\mathrm{AFz}$ and $\mathrm{FCz}$, respectively (Figure 2). All electrodes were mounted in an electrode cap placed on the subject's head, and conductive gel was applied in each of the electrode holes to keep impedance below $5 \mathrm{k} \Omega$.

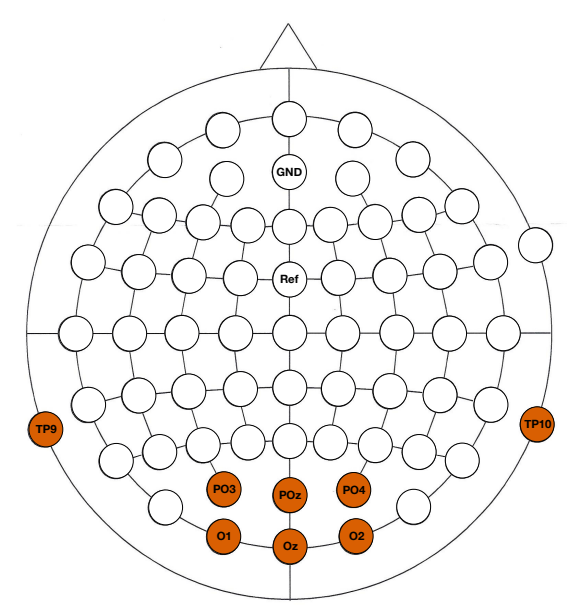

Fig. 2. EEG channels recorded during the experiment.

\section{Preprocessing}

The raw EEG recordings were re-referenced to the average of the mastoid signals and filtered between 4 and $20 \mathrm{~Hz}$, using a fourth-order zero-phase Butterworth filter. The filtered signals from the data collection session were then cut into 5 -second epochs, locked to the onset of the visual stimulation, while those from the main experiment into 12-second epochs. Prior to further analysis, the mastoid channels were removed such that only the six occipital channels remained. The analysis was implemented in Matlab (2017b).

\section{Spatiotemporal beamforming}

From the preprocessed EEG recordings, the temporal dynamics of the SSVEP during each trial was estimated using the spatiotemporal beamforming principle. This extension of the spatial beamforming algorithm estimates the presence of a targeted spatiotemporal activation pattern (i.e., template), and was first introduced for the analysis of the N400 event-related potential in the context of semantic priming [16], but has since then been used in BCI studies on the P300 event-related potential [21], [22], code-modulated visual evoked potential [23], [24], [25], motion-onset visual evoked potential [26] and SSVEP [27], [17] paradigms.
For the latter paradigm, the algorithm has also been shown effective in online [28] and invasive [29] settings. Given that the three stimulation frequencies used in this study elicit different temporal response patterns, three beamformers were built using the EEG recordings of the data collection session.

To construct the beamformer for frequency $f$, first, the epochs corresponding to the trials where the gazed target was flickering at frequency $f$ were isolated and cut into consecutive, non-overlapping segments $\mathbf{S}_{f} \in$ $\mathbb{R}^{m \times n \times k}$, where $m$ is the number of channels, $n$ the length of one period of frequency $f$, and $k$ the number of extracted segments. Next, all segments in $\mathbf{S}_{f}$ were averaged to obtain the activation pattern $\mathbf{A}_{f} \in \mathbb{R}^{m \times n}$. The covariance matrix $\boldsymbol{\Sigma}_{f} \in \mathbb{R}^{(m n) \times(m n)}$ was estimated from $\mathbf{T}_{f} \in \mathbb{R}^{k \times(m n)}$, a matrix whose rows correspond to the individual concatenated segments in $\mathbf{S}_{f}$. Finally, the beamformer $\mathbf{w}_{f} \in \mathbb{R}^{(m n) \times 1}$ for frequency $f$ is calculated as the Linearly-Constrained Minimum-Variance (LCMV) beamformer with constraint $\mathbf{a}_{f}^{\top} \mathbf{w}_{f}=1$ as follows:

$$
\mathbf{w}_{f}=\frac{\boldsymbol{\Sigma}_{f}^{-1} \mathbf{a}_{f}}{\mathbf{a}_{f}^{\top} \boldsymbol{\Sigma}_{f}^{-1} \mathbf{a}_{f}},
$$

where $\mathbf{a}_{f} \in \mathbb{R}^{1 \times(m n)}$ is vector containing the concatenated rows of $\mathbf{A}_{f}$.

\section{E. Estimating the temporal dynamics}

In previous SSVEP-BCI studies that relied on the spatiotemporal beamformer, a trial was classified by cutting the corresponding recording into consecutive, non-overlapping segments whose length equals the period of frequency $f$, averaging the extracted segments and applying beamformer $\mathbf{w}_{f}$, to obtain an estimate $y_{f} \in \mathbb{R}$ for each considered frequency $f$ [17]. The frequency yielding the highest estimate was then selected as winner. However, applying the beamformer to the average of the segments is mathematically equivalent to applying the beamformer to individual segments and averaging the obtained estimates:

$$
\left(\frac{1}{k} \sum_{j=1}^{k} \mathbf{s}_{j}\right) \mathbf{w}=\frac{1}{k}\left(\sum_{j=1}^{k} \mathbf{s}_{j} \mathbf{w}\right),
$$

where $\mathbf{s}_{j} \in \mathbb{R}^{1 \times(m n)}$ represents a vector containing the concatenated rows of segment $j$.

The latter procedure provides estimates for subsequent segments and thus allows for an investigation of the temporal dynamics of the SSVEP response.

\section{F. Classification}

In addition to the analysis of the temporal dynamics, we also aimed to predict the gazed target, for BCI purposes. To predict the row and column coordinates of the gazed target from an individual trial, the three beamformers (one for each of the three unique stimulation frequencies) were applied to obtain their temporal response dynamics during that trial (Figure 3), using 
the approach detailed in previous section. From the three temporal traces, the one having the highest average value was considered the winner (cf., equation 2), which in turn determined the row-coordinate of the predicted target. Next, the temporal dynamics of the winning frequency was isolated and used to predict the column of the gazed target. Every $500 \mathrm{~ms}$, the stimulation profile of one of the columns exhibited a transient modification, which is reflected in the isolated temporal dynamics. Thus, by detecting the transient in the temporal response dynamics, the gazed column can be identified.

For each transient stimulus modification during the considered trial, the activations of the respective beamformer (i.e., corresponding to the winning frequency) before and after the onset of the modification were extracted based on a weighted average of the response dynamics in a $500 \mathrm{~ms}$ window pre-onset and $500 \mathrm{~ms}$ window post-onset, respectively. The weights $\hat{\mathbf{b}}$ used in the average were linearly increased, favouring the later samples to avoid influence of the transitory period, either from a previous modification in case of the pre-onset window or the current modification in case of the postonset window, as follows: $\hat{\mathbf{b}}=\frac{\mathbf{b}}{|\mathbf{b}|}$, where $\mathbf{b}=[1 . . c]$, $c$ is the number of samples in each $500 \mathrm{~ms}$ window, which depends on the frequency, and $|\mathbf{b}|$ is the norm of vector $\mathbf{b}$. Next, for each of the four columns, the average post-onset activation was obtained as the mean of all post-onset activations of the said column. In addition, the average modulation for each column was calculated by subtracting the post-onset activations of the said column from their respective pre-onset activations and by taking the mean of those differences. Of the columns that had a negative post-onset activation, the one with the largest modulation was indicated as winning column. In case none of the post-onset activations was negative, the column with the smallest (i.e., closest to zero) postonset activation was identified as winning column.

This procedure was repeated for each trial from the main experimental sessions and for an increasing number of transient stimulation changes. Note that no cross-validation was needed, since the beamformers were trained on the data from the data collection session.

\section{RESULTS}

\section{A. Temporal dynamics}

Figure 3 shows the temporal dynamics of the three beamformers for one trial from the interruption- and phase reversal sessions. In both cases, only one trace exhibits high values (i.e., $\geq 1$ ) for a prolonged time while the other traces fluctuate around zero. The trace having the higher value represents the frequency the subject is gazing at (11 and $13 \mathrm{~Hz}$ for the interruption and phase-reversal case, respectively). In both subfigures, the value diminishes when the first column exhibits a change in its stimulation profile (indicated by the vertical lines with number 1 ), and returns to a high value when this column regains its original stimulation profile.
When the other columns exhibit a stimulus change, the value remains high. For the phase-reversal variant, the difference between the values during regular and modified stimulation is considerably larger (from $\geq 1$ to approximately -1 ), while for the interruption variant the output of the beamformer tailored to the $11 \mathrm{~Hz}$ flicker only reduces from $\geq 1$ to approximately zero. When the first column regains its original stimulation profile, the values of the respective beamformers return to a high state, indicating that the spatiotemporal pattern on which the beamformer was trained is present in the segments.

\section{B. BCI}

The row coordinate of the gazed target is deduced from the beamformer having the highest average output, and the column coordinate by detecting when the output from the respective beamformer stops being high. Both a stimulation interruption and a phase-reversal modification were tested, and the accuracy of target selection for both variations are shown in Figure 4. In both variations, targets can be selected with a median accuracy of $95 \%$ after 5 stimulus modifications. However, when reducing the number of modifications, there is a significant difference between both variants, with the phase-reversal modification maintaining a median accuracy of $88.3 \%$ for one stimulus modification while the interruption variant only reaches $73.3 \%(p<0.05$, Wilcoxon signed rank test).

\section{DISCUSSION}

In this study, we described how the spatiotemporal beamformer can be used to investigate the temporal dynamics of the SSVEP response. We showed that the temporal dynamics not only reflects the gazed frequency but also modifications in the stimulation profile, which we exploited to accurately detect the gazed target on single-trial basis.

Of the two stimulation profile modifications we tested, the phase-reversal pattern elicits the strongest anomaly and therefore yields the most accurate decoding performance when considered in a BCI context. The reason why anomalies in temporal dynamics can be reliably detected is because the spatiotemporal beamformer relies on a well-defined spatiotemporal template, elicited by (continuous) flickering stimulation. Given that the phase of the SSVEP has a stable intra-trial relationship with the phase of stimulation, reversing the phase of the stimulation also results in a reversal of the phase of the response. While the linear constraint in the LCMV beamformer enforces a value of 1 when the recordings are in agreement with the template, when the phase is reversed, the recordings will map to a value of -1 , leading to a larger anomaly compared to when the pattern is merely absent, such as in the case of the interruption profile. This phenomenon can clearly be seen in the temporal dynamics (figure 3), and can also 

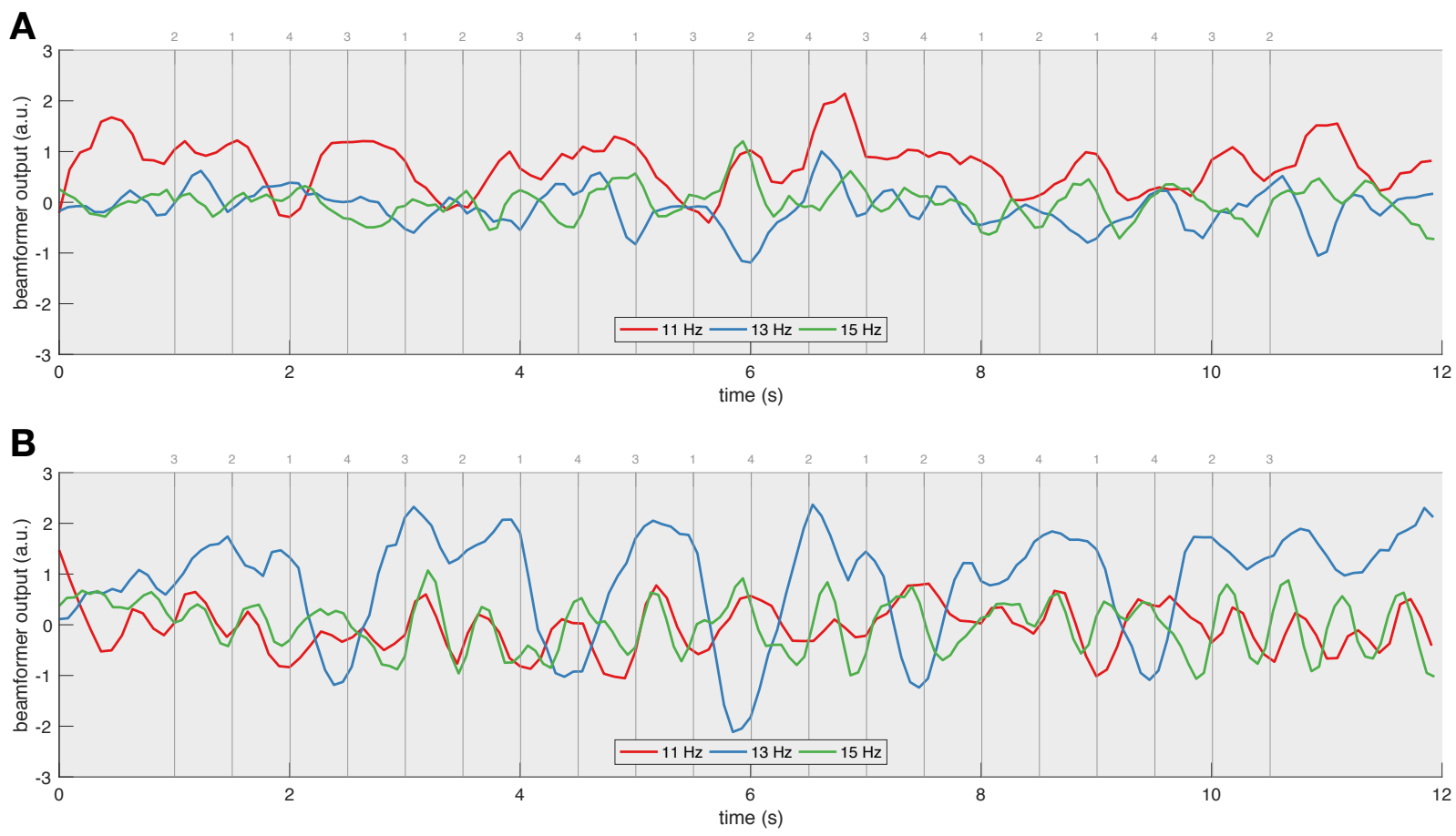

Fig. 3. Temporal dynamics of the SSVEP during one trial of subject 1 (A) with stimulation interruption (B) with phase reversals. The grey vertical lines indicate the onsets of the transient stimulation modifications and the numbers at the top of each line indicate which column exhibited this modification (cf. Figure 1C and D).

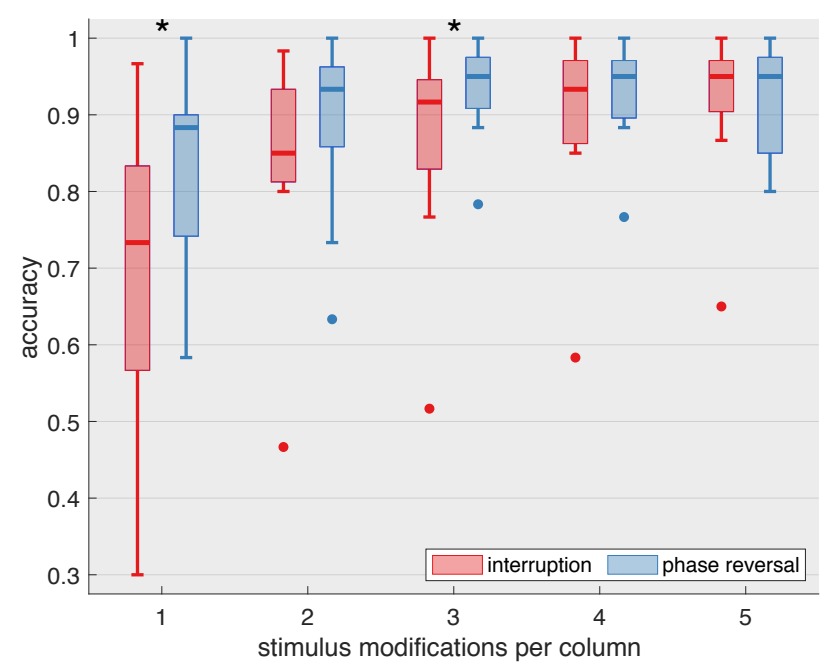

Fig. 4. Accuracy of target detection with respect to the number of stimulus modifications per column. Boxplots summarise the accuracies of the subjects and the dots represent outlier values.

accurately be detected on a single-trial basis using a simple algorithm. While not explored in this study, the temporal dynamics of the beamformer outputs could also be used to detect gaze switches from one frequency to another, or in paradigms that encode targets using a succession of different frequencies [30].

Given that stimulation variations are reflected by the beamformer outputs, one could use this feature to detect the gazed target in the context of visual BCI.
As the phase-reversal profile elicits more prominent anomalies, these are easier to detect, resulting in an impressive $88.3 \%$ accuracy on a single-trial level. It is worth noting that the detection of the intended target on a single-trial level is challenging. With the P300 paradigm, low single-trial accuracies are being reported $(<70 \%[21])$, even when using advanced classifiers [31] and stimulus optimizations that elicit additional EEG components [32], [33]. The purpose of this manuscript is not to compete with studies dedicated to maximize BCI performance. The classification approach used in our study is rather simple and could be improved using more advanced decoding techniques. Our goal was to show the capabilities of the spatiotemporal beamformer for the analysis of the temporal dynamics, which could spark novel, possibly more user-friendly, stimulation paradigms.

Despite the impressive performance of SSVEP-based $\mathrm{BCI}$ reported in the literature, considerable work is still needed with respect to asynchronous (i.e., non-cued) settings [13] and transfer learning. For the latter, data from a population of subjects could be used to obtain an accurate population model, thereby avoiding training sessions. One important challenge is phase variability across subjects [27]. Also in our future work, we will explore this topic.

\section{References}

[1] François-Benoît Vialatte, Monique Maurice, Justin Dauwels, and Andrzej Cichocki, "Steady-state visually evoked poten- 
tials: focus on essential paradigms and future perspectives," Progress in neurobiology, vol. 90, no. 4, pp. 418-438, 2010.

[2] Xiaogang Chen, Yijun Wang, Masaki Nakanishi, Xiaorong Gao, Tzyy-Ping Jung, and Shangkai Gao, "High-speed spelling with a noninvasive brain-computer interface," Proceedings of the national academy of sciences, vol. 112, no. 44, pp. E6058E6067, 2015.

[3] Ola Friman, Thorsten Luth, Ivan Volosyak, and Axel Graser, "Spelling with steady-state visual evoked potentials," in 2007 3rd International IEEE/EMBS Conference on Neural Engineering. IEEE, 2007, pp. 354-357.

[4] Nikolay Chumerin, Nikolay V Manyakov, Marijn van Vliet, Arne Robben, Adrien Combaz, and Marc M Van Hulle, "Steady-state visual evoked potential-based computer gaming on a consumer-grade eeg device," IEEE transactions on computational intelligence and ai in games, vol. 5, no. 2, pp. 100-110, 2013.

[5] Jonathan R Wolpaw, Niels Birbaumer, Dennis J McFarland, Gert Pfurtscheller, and Theresa M Vaughan, "Brain-computer interfaces for communication and control," Clinical neurophysiology, vol. 113, no. 6, pp. 767-791, 2002.

[6] Mikhail A Lebedev and Miguel AL Nicolelis, "Brain-machine interfaces: From basic science to neuroprostheses and neurorehabilitation," Physiological reviews, vol. 97, no. 2, pp. 767-837, 2017.

[7] Adrien Combaz, Camille Chatelle, Arne Robben, Gertie Vanhoof, Ann Goeleven, Vincent Thijs, Marc M Van Hulle, and Steven Laureys, "A comparison of two spelling brain-computer interfaces based on visual p3 and ssvep in locked-in syndrome," PloS one, vol. 8, no. 9, pp. e73691, 2013.

[8] Damien Lesenfants, Dina Habbal, Z Lugo, M Lebeau, Petar Horki, E Amico, Christoph Pokorny, F Gomez, A Soddu, Gernot Müller-Putz, et al., "An independent ssvep-based brain-computer interface in locked-in syndrome," Journal of neural engineering, vol. 11, no. 3, pp. 035002, 2014.

[9] Han-Jeong Hwang, Chang-Hee Han, Jeong-Hwan Lim, YongWook Kim, Soo-In Choi, Kwang-Ok An, Jun-Hak Lee, HoSeung Cha, Seung Hyun Kim, and Chang-Hwan Im, "Clinical feasibility of brain-computer interface based on steady-state visual evoked potential in patients with locked-in syndrome: Case studies," Psychophysiology, vol. 54, no. 3, pp. 444-451, 2017.

[10] Ivan Volosyak, "Ssvep-based bremen-bci interface-boosting information transfer rates," Journal of neural engineering, vol. 8, no. 3, pp. 036020, 2011.

[11] Nikolay V Manyakov, Nikolay Chumerin, and Marc M Van Hulle, "Multichannel decoding for phase-coded ssvep brain-computer interface," International journal of neural systems, vol. 22, no. 05, pp. 1250022, 2012.

[12] Chuan Jia, Xiaorong Gao, Bo Hong, and Shangkai Gao, "Frequency and phase mixed coding in ssvep-based braincomputer interface," IEEE Transactions on Biomedical Engineering, vol. 58, no. 1, pp. 200-206, 2011.

[13] Pablo F Diez, Agustina Garcés Correa, Lorena Orosco, Eric Laciar, and Vicente Mut, "Attention-level transitory response: a novel hybrid bci approach," Journal of Neural Engineering, vol. 12, no. 5, pp. 056007, Aug 2015.

[14] Minpeng Xu, Hongzhi Qi, Baikun Wan, Tao Yin, Zhipeng Liu, and Dong Ming, "A hybrid bci speller paradigm combining p300 potential and the ssvep blocking feature," Journal of neural engineering, vol. 10, no. 2, pp. 026001, 2013.

[15] Min Hye Chang, Jeong Su Lee, Jeong Heo, and Kwang Suk Park, "Eliciting dual-frequency ssvep using a hybrid ssvepp300 bci," Journal of neuroscience methods, vol. 258, pp. 104-113, 2016.

[16] Marijn Van Vliet, Nikolay Chumerin, Simon De Deyne, Jan Roelf Wiersema, Wim Fias, Gerrit Storms, and Marc M Van Hulle, "Single-trial erp component analysis using a spatiotemporal lcmv beamformer," IEEE Transactions on Biomedical Engineering, vol. 63, no. 1, pp. 55-66, 2016.

[17] Benjamin Wittevrongel and Marc M Van Hulle, "Spatiotemporal beamforming: A transparent and unified decoding approach to synchronous visual brain-computer interfacing," Frontiers in neuroscience, vol. 11, pp. 630, 2017.
[18] Nikolay V Manyakov, Nikolay Chumerin, Arne Robben, Adrien Combaz, Marijn van Vliet, and Marc M Van Hulle, "Sampled sinusoidal stimulation profile and multichannel fuzzy logic classification for monitor-based phase-coded ssvep brain-computer interfacing," Journal of neural engineering, vol. 10, no. 3, pp. 036011, 2013.

[19] David H Brainard and Spatial Vision, "The psychophysics toolbox," Spatial vision, vol. 10, pp. 433-436, 1997.

[20] Mario Kleiner, David Brainard, Denis Pelli, Allen Ingling, Richard Murray, Christopher Broussard, et al., "What's new in psychtoolbox-3," Perception, vol. 36, no. 14, pp. 1, 2007.

[21] Benjamin Wittevrongel and Marc M Van Hulle, "Faster p300 classifier training using spatiotemporal beamforming," International journal of neural systems, vol. 26, no. 03, pp. $1650014,2016$.

[22] Ruben Moermans, Benjamin Wittevrongel, and Marc M Van Hulle, "Mutual information-based electrode selection extended with prior knowledge for use in brain-computer interfacing," in 2018 IEEE International Conference on Bioinformatics and Biomedicine (BIBM). IEEE, 2018, pp. 2639-2644.

[23] Benjamin Wittevrongel, Elia Van Wolputte, and Marc M Van Hulle, "Code-modulated visual evoked potentials using fast stimulus presentation and spatiotemporal beamformer decoding," Scientific reports, vol. 7, no. 1, pp. 15037, 2017.

[24] Stavros I Dimitriadis and Avraam D Marimpis, "Enhancing performance and bit rates in a brain-computer interface system with phase-to-amplitude cross-frequency coupling: Evidences from traditional c-vep, fast c-vep, and ssvep designs," Frontiers in neuroinformatics, vol. 12, 2018.

[25] Zahra Shirzhiyan, Ahmadreza Keihani, Morteza Farahi, Elham Shamsi, Mina GolMohammadi, Amin Mahnam, Mohsen Reza Haidari, and Amir Homayoun Jafari, "Introducing chaotic codes for the modulation of code modulated visual evoked potentials (c-vep) in normal adults for visual fatigue reduction," PLOS ONE, vol. 14, no. 3, pp. 1-29, 03 2019.

[26] Arno Libert, Benjamin Wittevrongel, and Marc Van Hulle, "Evaluation of hybrid motion-onset and p300 target encoding for brain-computer interfacing," in Salzburg Mind-Brain Annual Meeting, Date: 2018/07/12-2018/07/13, Location: Salzburg, 2018.

[27] Benjamin Wittevrongel and Marc M Van Hulle, "Frequencyand phase encoded ssvep using spatiotemporal beamforming," PloS one, vol. 11, no. 8, pp. e0159988, 2016.

[28] Benjamin Wittevrongel and Marc M Van Hulle, "Hierarchical online ssvep spelling achieved with spatiotemporal beamforming," in 2016 IEEE Statistical Signal Processing Workshop (SSP). IEEE, 2016, pp. 1-5.

[29] Benjamin Wittevrongel, Elvira Khachatryan, Mansoureh Fahimi Hnazaee, Flavio Camarrone, Evelien Carrette, Leen De Taeye, Alfred Meurs, Paul Boon, Dirk Van Roost, and Marc M Van Hulle, "Decoding steady-state visual evoked potentials from electrocorticography," Frontiers in neuroinformatics, vol. 12, pp. 65, 2018.

[30] Yangsong Zhang, Peng Xu, Tiejun Liu, Jun Hu, Rui Zhang, and Dezhong Yao, "Multiple frequencies sequential coding for ssvep-based brain-computer interface," PloS one, vol. 7, no. 3, pp. e29519, 2012.

[31] Nader N Nashed, Seif Eldawlatly, and Gamal M Aly, "A deep learning approach to single-trial classification for p300 spellers," in 2018 IEEE 4th Middle East Conference on Biomedical Engineering (MECBME). IEEE, 2018, pp. 11-16.

[32] Jing Jin, Brendan Z Allison, Tobias Kaufmann, Andrea Kübler, Yu Zhang, Xingyu Wang, and Andrzej Cichocki, "The changing face of p300 bcis: a comparison of stimulus changes in a p300 bci involving faces, emotion, and movement," PloS one, vol. 7, no. 11, pp. e49688, 2012.

[33] Jing Jin, Brendan Z Allison, Xingyu Wang, and Christa Neuper, "A combined brain-computer interface based on p300 potentials and motion-onset visual evoked potentials," Journal of neuroscience methods, vol. 205, no. 2, pp. 265-276, 2012. 